\title{
Thoracic ultrasound for the diagnosis of pneumonia in adults: a meta-analysis
}

\author{
Thomas Berlet(10
}

\section{Letter to the Editor Thomas Berlet}

I read with interest the recent meta-analysis by Chavez and colleagues that was published in Respiratory Research, addressing the diagnostic performance characteristics of thoracic ultrasonography (TUS) in the diagnosis of pneumonia in adults. Favourable results for the diagnostic accuracy were reported, with calculated pooled sensitivities and specificities of $94 \%$ and $96 \%$, respectively [1].

When I reviewed the original data that the metaanalysis by Chavez and colleagues was based upon, I detected a number of issues that I wish to comment on. The authors analysed ten studies [2-11]. Six of these were performed in patients who were admitted to emergency departments or medical wards and who presented with signs and symptoms suggestive of pneumonia $[2,6$, 7, 9-11]. The study by Benci et al. enrolled 80 patients; 23 of these were diagnosed with interstitial pneumonia that could not be visualised with TUS [2]. The authors chose to exclude these patients from their calculation of diagnostic accuracy and focussed on the diagnosis of "alveolar pneumonia" instead. Therefore, the overall sensitivity of TUS for the diagnosis of any type of pneumonia was $61.7 \%$, and not $100 \%$, as cited by Chavez and colleagues [1]. The study by Parlamento and colleagues reported the sensitivity, but not the specificity, of TUS [6]. However, because normal TUS patterns were found in only 10 out of 17 patients who did not suffer from pneumonia, the specificity should have been calculated as $58.8 \%$, and not $100 \%$, as assumed by Chavez and colleagues [1]. The study by Reissig and colleagues enrolled 362 patients [9]. These authors chose to exclude equivocal TUS results from the calculation of diagnostic accuracy. By including these equivocal TUS findings in the calculation of diagnostic accuracy, sensitivity falls from $93.4 \%$ to $92.1 \%$, and specificity from $97.7 \%$ to $95.5 \%$. I

Correspondence: thomas.berlet@hospital-lingen.de

Inselspital/Bern University Hospital, Department of Intensive Care Medicine, 3010 Bern, Switzerland believe that the data from the above-mentioned three studies should have been critically reviewed and adjusted prior to inclusion in the meta-analysis.

Chavez and colleagues included four studies that were performed in critically ill patients in intensive care units who suffered from a variety of respiratory conditions [3-5, 8]. I am concerned that Chavez and colleagues failed to appreciate the fact that three out of four of these studies investigated the diagnostic accuracy of TUS for alveolar consolidation of any aetiology, rather than for the diagnosis of pneumonia.

Lichtenstein and colleagues studied 32 acute respiratory distress syndrome patients; 22 of these were diagnosed with pneumonia [3]. In another study, Lichtenstein and colleagues investigated 60 patients [4]. The inclusion criterion in this study was "exploration of chest pain or severe thoracic disease". Sixteen patients were diagnosed with pneumonia. While reporting high sensitivity and specificity of TUS for the detection of alveolar consolidation compared with computed tomography in both studies, Lichtenstein and colleagues did not differentiate between pneumonia patients and non-pneumonia patients. Xirouchaki and colleagues studied 42 patients [8]. While an admission diagnosis of sepsis or multiple organ dysfunction syndromes was present in 18 patients, no patient was diagnosed with pneumonia in their study. I believe that the results of these three studies should have been excluded from the meta-analysis because of methodological shortcomings.

A re-run of the meta-analysis by Chavez and colleagues [1], following correction of the data for sample size, sensitivity, and specificity in three studies $[2,6,9]$, and exclusion of those studies that failed to involve [8] or clearly identify pneumonia patients $[3,4]$ was performed, using Meta-DiSc V1.4 software (Unidad de Biostadística, Hospital Universitario Ramón y Cajal, Madrid, Spain). This analysis yielded significantly lower results for sensitivity and specificity of TUS for pneumonia than the initial meta-analysis by Chavez and colleagues [1] (Table 1). 
Table 1 Sensitivity and specificity table of thoracic ultrasonography for pneumonia

\begin{tabular}{|c|c|c|c|c|c|}
\hline Study & Sample size & Sensitivity & $95 \% \mathrm{Cl}$ & Specificity & $95 \% \mathrm{Cl}$ \\
\hline Benci et al. [2] & 80 & 0.62 & $0.48-0.74$ & 1.00 & $0.93-1.00$ \\
\hline Lichtenstein et al. [5] & 260 & 0.89 & $0.80-0.95$ & 0.9 & 0.97 \\
\hline Parlamento et al. [6] & 49 & 0.97 & $0.84-1.00$ & 0.59 & $0.33-0.82$ \\
\hline Cortellaro et al. [7] & 120 & 0.99 & $0.94-1.00$ & 0.95 & 0.83-0.99 \\
\hline Reissig et al. [9] & 362 & 0.92 & $0.88-0.95$ & 0.96 & $0.90-0.98$ \\
\hline Testa et al. [10] & 67 & 0.94 & $0.90-0.99$ & 0.85 & $0.68-0.95$ \\
\hline Unluer et al. [11] & 72 & 0.96 & $0.82-1.00$ & 0.84 & $0.7-0.94$ \\
\hline Pooled & 1010 & 0.9 & $0.87-0.92$ & 0.92 & $0.89-0.94$ \\
\hline Inconsistency $\left.\right|^{2}$ & & $88.1 \%$ & & $77.5 \%$ & \\
\hline
\end{tabular}

$\mathrm{Cl}$ is "confidence interval"

Since Chavez and colleagues performed their metaanalysis, another two clinical studies of the diagnostic accuracy of TUS for pneumonia have been published by Bourcier et al. [12], and Berlet et al. [13]. Onehundred forty-four [12], and 32 [13] patients were studied, raising the overall sample size to 1186 . If the results of these studies are added to the meta-analysis, sensitivity and specificity for the diagnosis of pneumonia using TUS rises to $91 \%$ (95\% confidence interval: 89-93\%) and specificity falls to $89 \%$ (95 \% confidence interval: 86-92\%). Heterogeneity between studies remains high, as reflected by high the inconsistency score. (Fig. 1).

\section{Conclusions}

Re-analysis of the results of studies of the use of diagnostic ultrasonography for pneumonia confirms that TUS is a useful tool for the diagnosis of the inflammatory consolidation of pneumonia. However, further research is required to improve the diagnostic accuracy of TUS in the diagnosis of pneumonia in adults.

\section{Authors response}

Miguel A Chavez, Laura E Ellington, Robert H Gilman, William Checkley

Over the last decade, there have been a growing number of research studies that evaluated the role of lung ultrasound for the diagnosis of pneumonia in adults [1]. Many of these studies preceded the development of standardized guidelines in sonographic methods and terminology [14], which may have contributed to heterogeneity in findings reported in our meta-analysis [1]. Differences in opinions about interpretation of findings may also affect how these studies are summarized. We appreciate Berlet's concerns about potential differences between our analysis and his revised analysis, and take this opportunity to reiterate more explicitly our choices. Moreover, we also demonstrate that our choices or the addition of new information from recently published studies had an overall small effect on the estimates of diagnostic accuracy. First, Berlet states that we chose to exclude participants with interstitial pneumonia from our calculations in the study by Benci et al. [2]. However,

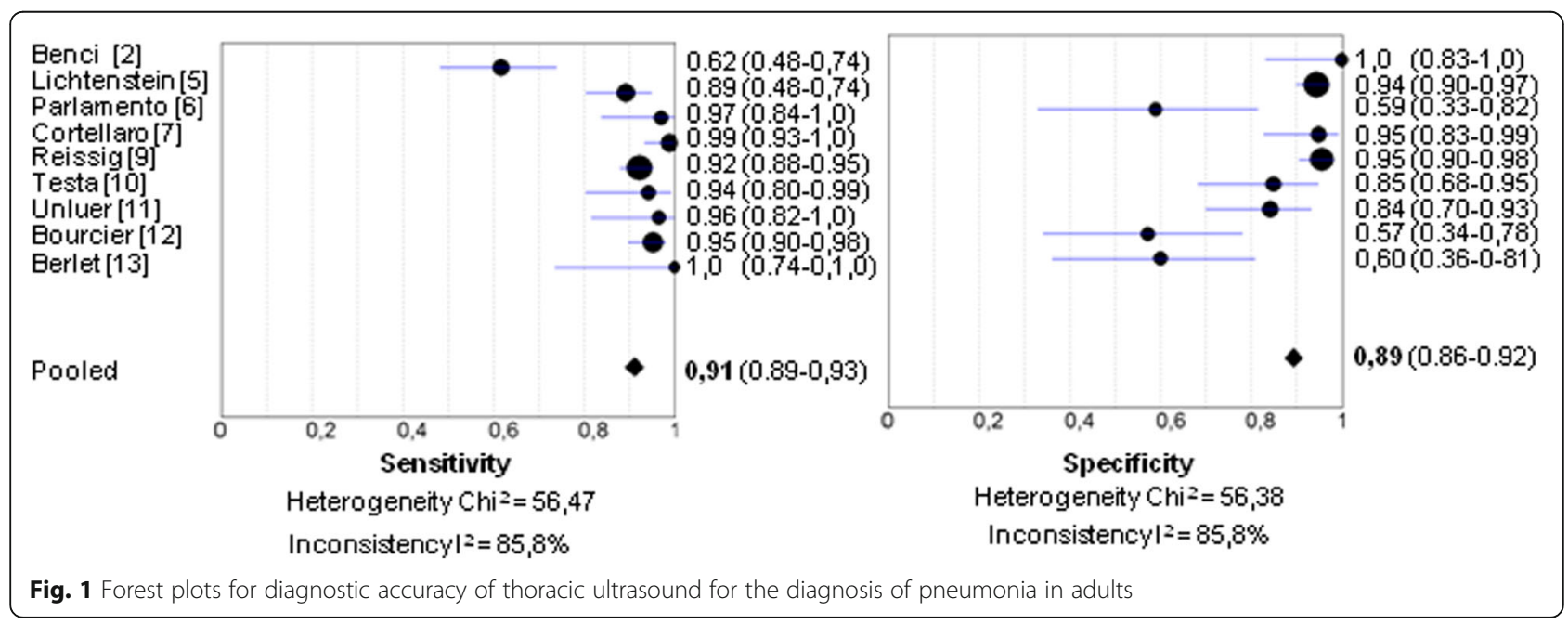



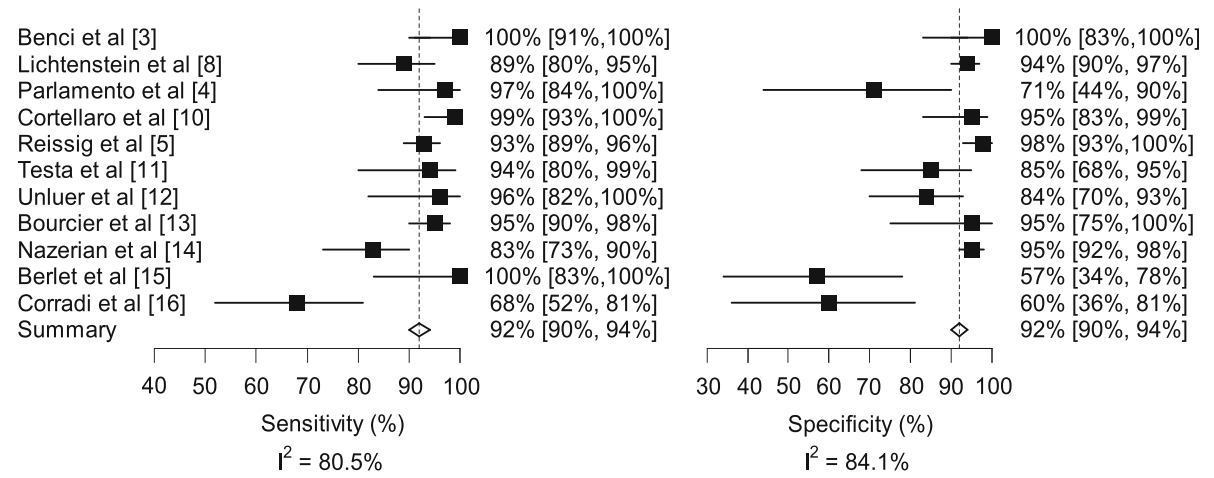

Fig. 2 Revised forest plots for diagnostic accuracy of lung ultrasound for the diagnosis of pneumonia

given that Benci et al. did not include interstitial findings by lung ultrasound within their methods [2], we decided to include only participants with either normal or lobar pneumonia by CXR in our analysis [1].

Second, we agree with Berlet that the numbers contributed by the study of Parlamento et al. could be revised [6]. Specifically, Parlamento et al. found that 7 out of 17 participants who did not have pneumonia had an abnormal ultrasound. Of those, Parlamento et al. were able to rule out infectious causes of consolidation in two participants by examining the air bronchogram characteristics or by the absence of air bronchograms. Although Parlamento et al. did not explicitly discuss specific songraphic findings for the remaining five participants with alveolar-interstitial syndrome, and were implicitly considered as a negative ultrasound for pneumonia [6]. Assuming that those five participants were indeed false positives, the revised estimate of specificity would be $95 \%$ (95 \% CI 94 \%-97 \%).

Third, we agree with Berlet that equivocal ultrasound results (1.7 \%) may affect estimates reported by Reissig et al. [9] and we could have reported this discrepancy in more detail. However, we did not consider this necessary since Reissig et al. [9] had already provided a detailed discussion about this point in their published paper. Moreover, although Reissig et al. had expert sonographers who performed the ultrasound, and in our own discussion we further emphasize that sonographer expertise is a critical element in assessing diagnostic accuracy.

Fourth, Berlet raises important concerns regarding studies performed in critically ill patients in intensive care units $[3-5,8]$ that were not directly addressed by our study [1]. Diagnostic accuracy in these studies was calculated for alveolar consolidation of any etiology including pneumonia, except for one study by Lichtenstein et al. [5] that specifically studied pneumonia. We agree with Berlet that the results of these studies $[3,4,8]$ have some methodological shortcomings. In our systematic review, we presented subgroup analyses confirming that when we excluded all studies conducted in intensive care units, sensitivity was $95 \%$ (95\% CI $93 \%-97 \%$ ) and specificity was $94 \%$ (95\% CI $91 \%-97 \%$ ) for the remaining studies [2, 6, 7, 9-11].

Finally, since the publication of our meta-analysis, at least four new studies have been published $[12,13,15,16]$. When we analyzed information from these recent studies $[13,15,16]$, our revised estimates yielded an overall sensitivity of $92 \%$ (95\% CI $90 \%-94 \%)$ and specificity of $92 \%$ (95\% CI 90 \%-94\%; Fig. 2), with an AUC of 0.97 (95\% CI, 0.95 to 0.99 ), which is similar in range to values reported by our original analyses [1]. None of the revised estimates, however, affects our final conclusion that lung ultrasound appears to be a reasonable alternative to chest radiography for the diagnosis of pneumonia in adults. However, we agree that further research is needed to assess the validity of lung ultrasound in a variety of settings and degrees of sonographer expertise.

\section{Abbreviations}

Cl: Confidence interval; TUS: Thoracic ultrasonography.

\section{Competing interests}

The author declares that he has no competing interests.

\section{Author's contribution}

TB conducted the review of published papers, the analysis and interpretation of data, and wrote the manuscript.

\section{Acknowledgements}

The author received solely departmental funding.

Received: 26 March 2015 Accepted: 1 July 2015

Published online: 25 July 2015

\section{References}

1. Chavez MA, Shams N, Ellington LE, Naithani N, Gilman RH, Steinhoff MC, et al. Lung ultrasound for the diagnosis of pneumonia in adults: a systematic review and meta-analysis. Respir Res. 2014;15:50.

2. Benci A, Caremani M, Menchetti D, Magnolfi AL. Sonographic diagnosis of pneumonia and bronchopneumonia. Eur J Ultrasound. 1996;4:169-76. BIBLIOGRAPHY $\backslash 2055$.

3. Lichtenstein D, Goldstein I, Mourgeon E, Cluzel P, Grenier P, Rouby JJ. Comparative diagnostic performances of auscultation, chest radiography, 
and lung ultrasonography in acute respiratory distress syndrome. Anesthesiology. 2004;100:9-15.

4. Lichtenstein D, Lascols N, Mezière G, Gepner A. Ultrasound diagnosis of alveolar consolidation in the critically ill. Intensive Care Med. 2004;30:276-81.

5. Lichtenstein DA, Meziera GA. Relevance of lung ultrasound in the diagnosis of acute respiratory failure: the BLUE protocol. Chest. 2008;134:117-25.

6. Parlamento S, Copetti R, Di Bartolomeo S. Evaluation of lung ultrasound for the diagnosis of pneumonia in the ED. Am J Emerg Med. 2009;27:379-84.

7. Cortellaro F, Colombo S, Coen D, Duca PG. Lung ultrasound is an accurate diagnostic tool for the diagnosis of pneumonia in the emergency department. Emerg Med. 2012;19:19-23.

8. Xirouchaki N, Magkanas E, Vaporidi K, Kondili E, Plataki M, Patrianakos A, et al. Lung ultrasound in critically ill patients: comparison with bedside chest radiography. Intensive Care Med. 2001;37:1488-93.

9. Reissig A, Copetti R, Mathis G, Mempel C, Schule A, Zechner P, et al. Lung ultrasound in the diagnosis and follow-up of community-acquired pneumonia. Chest. 2012;4:965-72.

10. Testa A, Soldati G, Copetti R, Giannuzzi R, Portale G, Gentiloni-Silveri N. Early recognition of the 2009 pandemic influenza A (H1N1) pneumonia by chest ultrasound. Crit Care. 2013;16:R30.

11. Unluer E, Karagoz A, Senturk G, Karaman M, Olow K, Bayata S. Bedside lung ultrasonography for diagnosis of pneumonia. Hong Kong Am J Emerg Med. 2013;20:98.

12. Bourcier J, Paquet J, Seinger M, Gallard E, Redonnet J, Cheddadi F, et al. Performance comparison of lung ultrasound and chest $x$-ray for the diagnosis of pneumonia in the ED. Am J Emerg Med. 2014;32:115-8.

13. Berlet T, Etter R, Fehr T, Berger D, Sendhi P, Merz TM. Sonographic patterns of lung consolidation in mechanically ventilated patients with and without ventilator-associated pneumonia: a prospective cohort study. J Crit Care. 2015;30:327-33.

14. Volpicelli G, Elbarbary M, Blaivas M, Lichtenstein DA, Mathis G, Kirkpatrick AW, et al. International Liaison Committee on Lung Ultrasound (ILC-LUS) for International Consensus Conference on Lung Ultrasound (ICC-LUS): International evidence-based recommendations for point-of-care lung ultrasound. Intensive Care Med. 2012;38:577-91.

15. Nazerian P, Volpicelli G, Vanni S, Gigli C, Betti L, Bartolucci M, et al. Accuracy of lung ultrasound for the diagnosis of consolidations when compared to chest computed tomography. American J Emer Med. 2015;33:620-5.

16. Corradi F, Brusasco C, Garlaschi A, Paparo F. Quantitative analysis of lung ultrasonography for the detection of community-acquired pneumonia: a pilot study. Biomed Res Int. 2015;2015:868707.

\section{Submit your next manuscript to BioMed Central and take full advantage of:}

- Convenient online submission

- Thorough peer review

- No space constraints or color figure charges

- Immediate publication on acceptance

- Inclusion in PubMed, CAS, Scopus and Google Scholar

- Research which is freely available for redistribution 